\title{
Combining motility and bioluminescent signalling aids mate finding in deep-sea fish: a simulation study
}

\author{
Graeme D. Ruxton ${ }^{1, *}$, David M. Bailey ${ }^{2}$ \\ ${ }^{1}$ Division of Environmental \& Evolutionary Biology, Institute of Biomedical \& Life Sciences, University of Glasgow, \\ Glasgow G12 8QQ, UK \\ ${ }^{2}$ Marine Biology Research Division, Scripps Institution of Oceanography, University of California San Diego, \\ 9500 Gilman Drive, La Jolla, California 92093-0202, USA
}

\begin{abstract}
We present a model to estimate the mean time required for mate finding among deepsea fish as a function of motility and the extent of bioluminescent signalling. This model differs from those of previous works in 3 important ways by including (1) sex differences in motility, (2) a maximum detection range of bioluminescent signals derived from a recently published mechanistic model based on physical principles and the physiology of vision, and (3) a novel consideration of the likelihood of individuals passing within detection range only in the interval between flashes and hence, failing to detect the signaller. We argue that the flash rates required for effective detection are low, with rates of less than 1 per minute being entirely plausible, and that predation pressure may further encourage low flash rates. Further, even at high flash frequencies, the energetic cost of bioluminescent signalling is argued to be a trivial fraction of resting metabolic rates. Using empirically derived estimates for parameter values, we estimate that a female will be detected and reached by a male within 2 to $4 \mathrm{~h}$ of beginning to signal. Hence, we argue that mate finding may not seriously restrict reproductive success in species that can exploit this signalling system. We further argue that where male motility allows bioluminescent signalling, this may have some advantages over chemical-based signalling. Bioluminescent signalling may, therefore, be more important to mate finding in the deep sea (relative to chemical signals) than some previous works have suggested.
\end{abstract}

KEY WORDS: Bioluminescence $\cdot$ Mating $\cdot$ Predation $\cdot$ Mid-water fish $\cdot$ Olfaction

\section{INTRODUCTION}

Mate finding can be a challenging aspect of lifehistory for sexually reproducing species that live at low population densities. Deep-sea fish are likely to provide examples of this, given that the low food availability in the deep sea generally constrains species biomass to very low densities (Herring 2002). This paper will focus on 2 inter-related methods of mitigating this challenge in deep-sea fish: (1) motility and (2) bioluminescent signalling.

In the dark of the deep sea, in the absence of selfgenerated light, the distance at which one member of a species can detect the presence of another member of the same species visually is likely to be less than $1 \mathrm{~m}$
(Denton 1990, Johnsen \& Sosik 2003). Detection via the lateral line system is likely to be even more spatially constrained (Denny 1993). Underwater acoustic signals may be difficult to localise (Denny 1993). Hence, the 2 (non-exclusive) most commonly considered means by which longer-range detection could occur are via the emission and detection of either bioluminescent or chemical signalling. We follow Herring (2000) in focussing on the first of these.

Herring (2000) provides a recent summary of our understanding of the role of bioluminescent signalling as a means of mate detection in the deep sea. As he points out, there is no direct and unequivocal evidence that any deep-sea species uses bioluminescent signalling for this purpose. However, there are many 
large gaps in our understanding of the ecology of the deep sea (e.g. Herring 2002) and much circumstantial evidence that the bioluminescent organs that are widespread in deep-sea fish have a sexual function, a conclusion generally based on sexual dimorphism in the existence or size of the organs (see Herring 2000 for an overview). Herring (2000) estimates average nearest neighbour distances for a variety of deep-sea fish species of between 0.5 and $24 \mathrm{~m}$. He argues that his methods will tend to underestimate these distances, but even at face value, many of these distances are likely to be beyond the maximum detection range of bioluminescent flashes (which he estimates to be of the order of $10 \mathrm{~m}$ ). His conclusion from this comparison is that bioluminescent signals cannot be the primary means by which mate discovery is achieved, but rather that 'these signals are employed at intermediate ranges, once an initial contact (perhaps olfactory) has been made'. Whilst the scenario of long-range detection by chemical means followed up by closer range bioluminescent signalling is entirely plausible, we do not feel that Herring's arguments should lead us to conclude that such a scenario is inevitable, and hence that bioluminescence is destined to be only the handmaiden to chemical signalling.

Herring's arguments are most compelling if individuals are effectively motionless in the absence of having detected a signal from a potential mate. However, if the ecology of a species is such that individuals are in motion (relative to the water around them, either in search of food or mates), then average nearest neighbour distances do not tell the full story. When in motion, individuals may periodically come very close to each other as a result of their relative motion, even though on average they are often far away from each other. Hence, as Herring (2000) acknowledges, a full evaluation of the importance of bioluminescence for mobile animals like fish requires a dynamic model that considers temporal changes in the distances between individuals. This paper will present and explore such a model.

Herring (2000) pointed to previous work of Baird \& Jumper (1995) as providing a suitable methodology for studying the interaction rates of moving fish. This model assumes that both males and females move at random in 3 dimensions at an average individual speed $v$. Implicitly, this model considers midwater fish, far from any landmark features provided by the bottom. Hence, a stationary fish is stationary with respect to the water around it, although this water may experience large-scale bulk movement through oceanic circulation. As an individual moves through the water, it can be imagined as creating a tube around it, centred on the trajectory of the animal and with a constant radius $R$, which is the maximum distance at which indi- viduals can detect each other. If the focal individual's tube envelops another individual, then the focal individual detects that individual. This model can be used to produce a simple expression for the probability that the focal individual will have discovered another individual within some specified time. Baird \& Jumper (1995) used this to explore the likely importance of bioluminescent signalling for the mesopelagic hatchetfish Sternoptyx diaphana. Their most important finding was the highly non-linear relationship between detection probabilities and the maximum detection radius $R$. Here, we will take their methodology and develop it in a number of significant ways.

Baird \& Jumper (1995) assumed that both sexes moved, whereas game theory models of intra- and inter-sexual conflict in the evolution of mate-attraction signals suggest that only one sex is likely to signal and remain motionless, while the other sex explores the environment searching for these signals (Hammerstein \& Parker 1987, Bradbury \& Vehrencamp 1998). Hence, we will assume that females signal but remain motionless, with males searching for these signals.

Baird \& Jumper (1995) did not attempt to estimate the maximum detection range parameter $R$, but rather used a range from 1 to $10 \mathrm{~m}$. Since then, Warrant (2000) has published a simple model based on the physics of light detection, developed to give estimates of the distance at which the eyes of deep-sea fish could detect bioluminescent flashes. We will use this method to estimate appropriate values for this critical parameter.

Baird \& Jumper (1995) assume that if a male and female fish approach to within a distance less than $R$, then detection is certain. However, since bioluminescent signals are generally not constant but flash intermittently (Clark \& Hubbard 1959), it is possible that a male may pass within $R$ of the female in the time between flashes, and so fail to detect her. Hence, our model will include an extra term to represent this possibility. This will require an entirely novel model of the flash rate of females to be developed; such a model is presented in the next section.

\section{MODEL}

\section{A model of flash frequency}

For simplicity, we assume that males change direction sufficiently infrequently that we can consider their trajectories when passing close to the female to be a simple straight line. We are interested in the intersection of this trajectory with the detection zone around the female within which the male can detect flashes by the female. Again for simplicity, we will assume that this zone is a sphere centred on the 
female with a radius defined by the maximum distance at which the male can detect the female's flashes $R$. This very simple geometry assumes the female's signal radiates equally well in all directions, and the background against which it is viewed is also the same from all directions. Further, this assumption implicitly assumes that the interaction takes place in very deep water where sunlight penetration is negligible. Since the male's trajectory will be unaffected by the female until he detects a signal (which can only happen in the detection zone), we assume that the trajectories of males are orientated randomly with respect to the female and her detection zone (that is, they form a randomly orientated chord of the spherical detection zone). It is our requirement for a simple analytic form for the distribution of such chord lengths that has required us to assume a spherical geometry; the consequences of this assumption will be considered further in 'Discussion'. Let us assume that the female flashes at constant intervals with period $T$ between flashes. It is easy to see that such even spacing is the most effective way for the female to space her flashes so as to minimise the risk of males failing to detect her. A male travelling at speed $v$ will travel a distance $v T$ between flashes. Hence, trajectories within the detection zone of length $L$ greater than $v T$ guarantee detection.

Let us now consider the case where $L<v T$. If we imagine that the female flashes at time zero, if the male enters immediately afterwards, then he will travel through the detection zone and out again before the female flashes again. This is true for all entry times in the range $(0, T-[L / V])$, whereas, for all entry times in the range $(T-[L / V], T)$, the male is still in the detection zone when the female next flashes. We can make a similar argument for entry points between any 2 flashes in the female's sequence. We assume that the male will enter at a time that is entirely at random with respect to the female's sequence; thus, the probability of the male detecting the female is simply given by the fraction of possible entry times that lead to detection. From our arguments above, this is simply $L /(v T)$. Thus, the probability of a trajectory of length $L$ leading to detection $P(L)$ is given by:

$$
P(L)= \begin{cases}\frac{L}{v T} & L<v T \\ 1 & L \geq v T\end{cases}
$$

Jokisch et al. (2001) give an expression for the frequency distribution of randomly orientated chords across a sphere:

$$
f(L)=\frac{L}{2 R^{2}}
$$

The probability $A$ of a female attracting a male that enters her zone of detection is given by:

$$
A=\int_{0}^{2 R} P(L) f(L) \mathrm{d} L
$$

since the maximum chord length across any sphere is simply twice the radius.

If $2 R<v T$, then no chords through the sphere are large enough to guarantee detection, and so (using Eqs. 1 \& 2), we can evaluate Eq. (3) to give:

$$
A=\frac{4 R}{3 v T}
$$

For the case where $2 R \geq v T$, integration is slightly more involved (but still straightforward) and yields the expression:

$$
A=1-\left(\frac{1}{12}\right)\left(\frac{v T}{R}\right)^{2}
$$

It is trivial to show that both Eqs. (4) \& (5) give the same value $(2 / 3)$ in the boundary case where $2 R=v T$. We now use Eqs. (4) \& (5) to explore how the probability $A$ that a male passing through the female's detection zone actually detects the female, varies as a function of the values of parameters $V, T$ and $R . R$ is the maximum distance at which males can detect females' flashes. This can be estimated from the model of Warrant (2000):

$$
N=\left(\frac{E D^{2}}{16 R^{2}}\right) \exp (-\alpha R)
$$

where $N$ is the minimum number of photons required to enter the eye in order to facilitate detection, $E$ is the number of photons produced by a flash, $D$ is the diameter of the pupil of the eye and $\alpha$ is the beam attenuation coefficient of the water. Warrant suggests the following values $N=5, E=10^{10}$ and $\alpha=0.05 \mathrm{~m}^{-1}$. These values seem reasonable: Mesenger \& Case (1990) artificially stimulated flashes in specimens of 15 species of deep-sea fish and measured peak flash intensities of $5 \times 10^{9}$ to $9 \times 10^{11}$ photons $\mathrm{s}^{-1}$, and flash durations ranging from 0.2 to $4 \mathrm{~s}$, suggesting that $E$ values around $10^{10}$ seem appropriate (although in situ measurement of naturally occurring flashes would be very welcome). The parameter $\alpha$ is relatively well-known and (although it varies with wavelength and properties of the water) $0.05 \mathrm{~m}^{-1}$ is a very typical value (e.g. Denton 1990, Johnsen et al. 2004). Assuming a high contrast of $50 \%$ (i.e. that the background is very dark), then $N=5$ fits with our understanding of the Rose-de Vries law of signal detection (Land \& Nilsson 2002). As an example fish, we take the mesopelagic hatchetfish Sternoptyx diaphana, considered in the model of Baird \& Jumper (1995); it has a standard length of $30 \mathrm{~mm}$ and a pupil diameter $D$ of $2 \mathrm{~mm}$. We consider this species for reasons of commonality with previous work, rather than because we consider it particularly representative or 
interesting. Baird \& Jumper suggest that reasonable search rates for this fish would be 6 to $60 \mathrm{~mm} \mathrm{~s}^{-1}$ (i.e. 0.2 to 2 body-lengths $\mathrm{s}^{-1}$ ). As a default value, we will adopt $15 \mathrm{~mm} \mathrm{~s}^{-1}\left(0.5\right.$ body-length $\left.\mathrm{s}^{-1}\right)$; that is, $V=$ $0.015 \mathrm{~m} \mathrm{~s}^{-1}$. Before presenting the full model of encounter rates between males and females, we will explore the behaviour of this submodel.

For these default values, we find (using Eq. 6) that the radius of detection $R$ is $15 \mathrm{~m}$. In comparison to this radius, even the high estimate of the male's travel speed is quite sedate, and so if the female flashes every $5 \mathrm{~min}$, the probability of a male entering the female's detection zone and detecting one of her flashes (calculated using Eqs. 4 to 6) is high (always $>85 \%$ for the range of speeds considered; see Fig. 1a). Even if the flash rate dropped to once every $30 \mathrm{~min}$ (1800 s), the

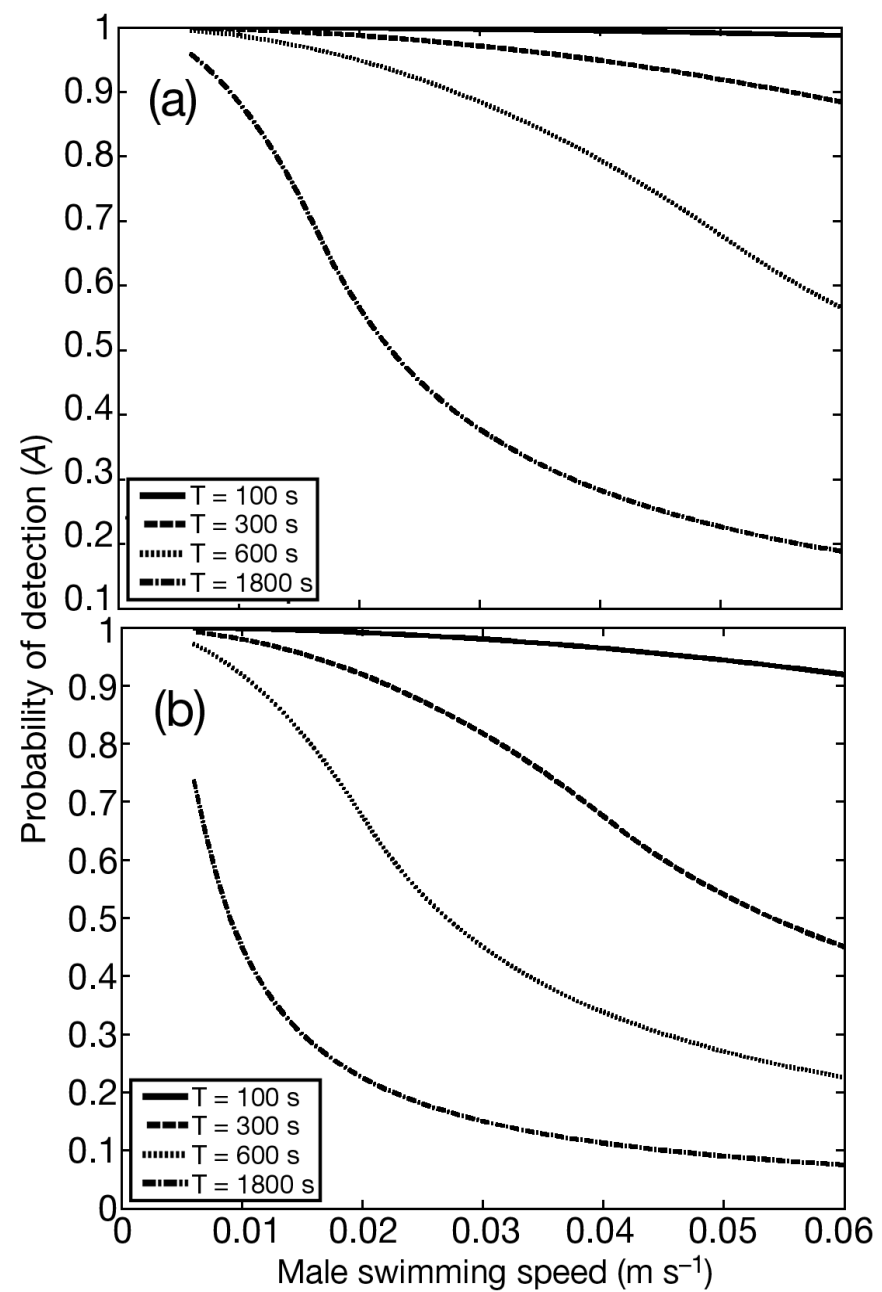

Fig. 1. Probability $(A)$ of the male detecting a female while crossing her detection zone, as a function of male swimming speed $v\left(\mathrm{~m} \mathrm{~s}^{-1}\right)$ and the period, in seconds, of the female's flashing $(T)$; calculated using Eqs. (4), (5) \& (6). In (a) $E=$ $10^{10}$ photons; in (b) $E=10^{9}$ photons. Other parameter values: $N=5 ; \alpha=0.05 \mathrm{~m}^{-1} ; D=0.002 \mathrm{~m}$ probability of detection is still relatively high providing the male's travel speed is below $2 \mathrm{~cm} \mathrm{~s}^{-1}$. This suggests that the female could afford to send out a rather less powerful signal. If we decrease $E$ by a factor of 10 to $10^{9}$, then $R$ drops to $6.1 \mathrm{~m}$. This smaller $R$ value reduces the average time it will take the male to swim through the detection zone. However, required flash rates for effective detection are still relatively modest, with a flash rate of once every $100 \mathrm{~s}$ still giving a success rate $>90 \%$ over all considered swimming speeds (see Fig. 1b).

We might imagine that flashing is energetically expensive for the females, and that there is a trade-off between flashing brightly (which would require a low flash rate to give a high $A$ value) or flashing more dimly (but compensating by flashing more frequently). We now explore this quantitatively. The majority of bioluminescent emission spectral maxima are in the light region ranging from 450 to $530 \mathrm{~nm}$ (Herring 1983). A photon of $500 \mathrm{~nm}$ wavelength has an energy content of $4 \times 10^{-19} \mathrm{~J}$. However, production of bioluminescent light is likely to be less than $100 \%$ efficient. It has been suggested that 6 molecules of ATP are required to generate a bioluminescent photon (Hastings \& Nealson 1981, Nunes-Halldorson \& Duran 2003). If $80000 \mathrm{~J}$ are required to form $1 \mathrm{~mol}$ of ATP (Alexander 1999), this suggests that production of a bioluminescent photon costs $8 \times 10^{-19} \mathrm{~J}$. Taking this latter figure, the power $P$ required to produce $E$ photons every $T$ seconds is given by:

$$
P=\frac{8 \times 10^{-19} E}{T}
$$

If we substitute our brightest flash $E=10^{10}$ and a rapid flash rate (equivalent say to $T=1 \mathrm{~s}$ ), this gives $P=$ $8 \times 10^{-9} \mathrm{~W}$. The wet weight of a $30 \mathrm{~mm}$ Sternoptyx diaphana is likely to be of the order of $4 \mathrm{~g}$ (Howell \& Krueger 1987, Mensinger \& Case 1997). Torres et al. (1979) measured resting metabolic rates in a number of midwater fishes, and obtained a representative value for a $4.2 \mathrm{~g}$ fish of $0.08 \mu \mathrm{l} \mathrm{O} \mathrm{g}_{2}$ wet weight ${ }^{-1} \mathrm{~h}^{-1}$. Using the conversion $1 \mathrm{ml} \mathrm{O}_{2}=19.4 \mathrm{~J}$ from Drazen (2002), this gives a metabolic rate of the order of $0.002 \mathrm{~W}$. If the metabolic rate of $S$. diaphana is similar to this, then our calculations above suggest that the energetic cost of bioluminescent signalling may be trivial. It is likely that a more substantial cost of flashing for females lies in alerting predatory fish to their presence. Again, we can use the model to explore the likely design consequences of this.

We can expect predators in general to be larger and have larger eyes than male conspecifics of the signalling females. Tunas are known predators of Sternoptyx diaphana (Kornilova 1990). If we take $1.5 \mathrm{~m}$ as the characteristic length of a tuna, then 
even a low cruising speed of 0.5 body lengths $\mathrm{s}^{-1}$ works out at $0.75 \mathrm{~m} \mathrm{~s}^{-1}, 50$ times the expected searching speed of Sternoptyx males. This fast speed should increase the likelihood of the predatory tuna failing to detect a female because it passes through the detection zone between flashes. However, this effect will be counteracted to some extent by the detection zone being bigger for predators than conspecific males because eye size will be larger in the predators. However, eye size increases relatively slowly with increasing size in fish (Howland et al. 2004) and a $1.5 \mathrm{~m}$ long tuna has a pupil diameter of around $2 \mathrm{~cm}$ (Nelson et al. 2001). Thus, the $D$ value for our notional predator is only 10 times that of the conspecific males. Hence, providing the flash rate is low (i.e. $T$ is high), we can have the situation illustrated in Fig. 2 where conspecific males $(D=2 \mathrm{~mm}, V$ $=0.015 \mathrm{~m} \mathrm{~s}^{-1}$ ) are very likely to detect a female if they enter the detection zone, whereas predators (because of their bigger eye sizes [ $D=20 \mathrm{~mm}$ ] do not fully compensate for their much greater speed $[\mathrm{V}=$ $0.75 \mathrm{~m} \mathrm{~s}^{-1}$ ]) are relatively likely to pass through the detection zone between flashes. This should lead to another evolutionary pressure for low flash rates by females. However, in order for females to minimise their predation risk, the key is likely to lie in minimising the time over which the signal is employed, as well as specific aspects of the signal design. To study this, we must turn to the full model of searching males.

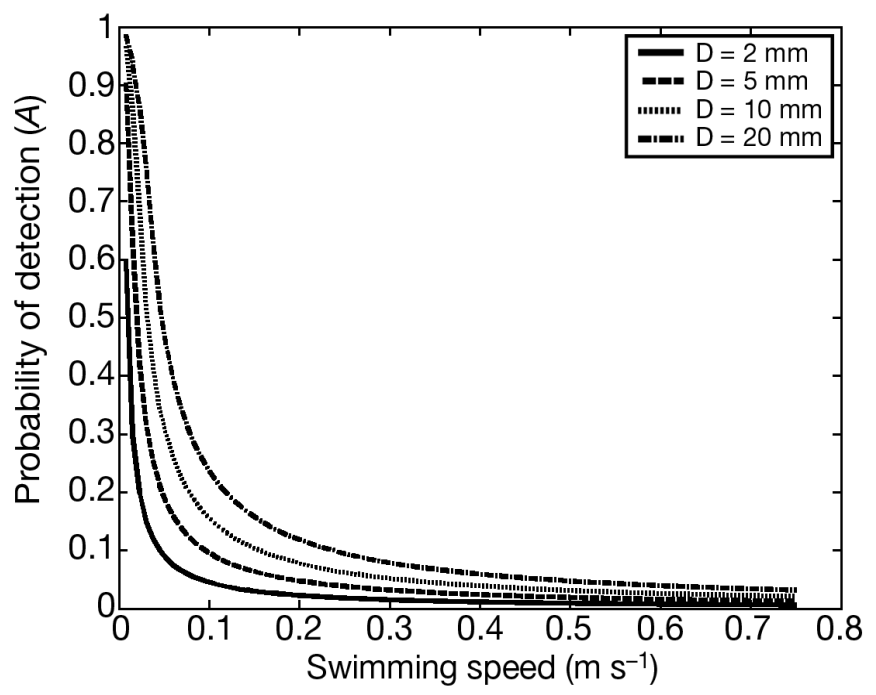

Fig. 2. Probability $(A)$ of a fish (either a male conspecific or a predator) detecting the female while crossing her detection zone, as a function of swimming speed $v\left(\mathrm{~m} \mathrm{~s}^{-1}\right)$ and pupil diameter $D$ (in mm); calculated using Eqs. (4), (5) \& (6). Other parameter values: $N=5 ; E=10^{10} ; \alpha=0.05 \mathrm{~m}^{-1} ; T=1800 \mathrm{~s}$

\section{A full model of the probability of mate finding within a specified time}

We assume that at some point (which we label time $t=0$ ), a female begins bioluminescent signalling. We assume that sexually mature males exist at a density $\rho$, and they move at average speed $v$, randomly searching for a signalling female. We assume that females are sufficiently rare that over the temporal and spatial scales of interest no significant depletion of males occurs due to pairing with females other than our focal individual, and so $\rho$ is a simple constant. The rate $Z$ at which males come within distance $R$ (given by Eq. 6) of our focal species is given simply by the rate at which the detection tubes sweep out new volume of the environment (e.g. Gerritsen \& Strickler 1977, Baird \& Jumper 1995):

$$
Z=\pi R^{2} \rho v
$$

This formulation assumes that the male avoids researching previously explored parts of the environment, which can be best achieved by having a low turning rate. However, only a fraction $A$ of encounters (given by either Eq. 4 or 5) leads to detection of the female, so the probability of at least 1 male discovering the female by some time $t$ after the commencement of signalling is given by:

$$
P(T)=1-\exp (-Z A t)
$$

or alternatively, the time $\tau$, required to obtain a fixed probability of detection $P^{*}$, is given by:

$$
\tau=\frac{-\ln \left(1-P^{*}\right)}{Z A}
$$

Again, we require estimates for a number of parameters. Baird \& Jumper (1995) suggest values (based on trawl samples) of 23 to 50 ind. $10^{6} \mathrm{~m}^{-3}$ (i.e. 2.3 to $5 \times 10^{-5} \mathrm{~m}^{-3}$ ) for the density of adult males $(\rho)$ in the species Sternoptyx diaphana, possibly dropping as low as 0.05 ind. $10^{6} \mathrm{~m}^{-3}$ for some other species. By nothing more than convention, we will fix $P^{*}$ at a $95 \%$ probability. Fig. 3 gives $\tau$ as a function of the density of adult males parameterised for $S$. diaphana, suggesting that for the estimated population densities, a female would need to signal for between 2 and $4 \mathrm{~h}$ in order to be $95 \%$ certain of attracting at least 1 male. In order to explore the generality of this prediction, we must first consider how the population density values used in Fig. 3 relate to the estimated average nearest neighbour distances used by Herring (2000). Herring (2000) used an equation derived by Mackie \& Mills (1983), which uses arguments based on the close packing of spheres, to give the following relation between population density $(\rho)$ and nearest neighbour distance $D_{N N}$ : 


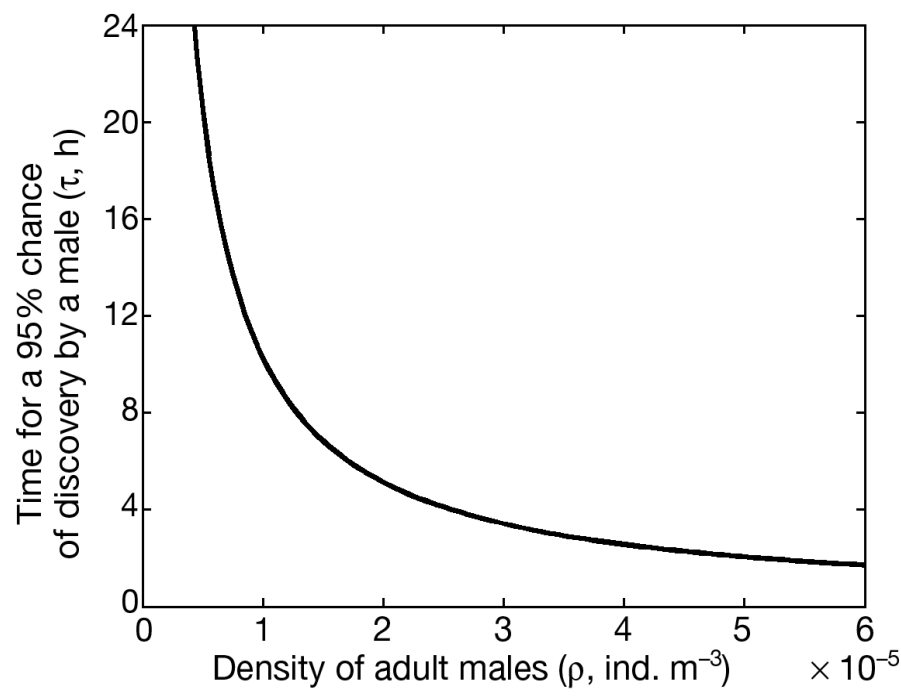

Fig. 3. Estimated time $(\tau)$ for which a female would have to signal to be $95 \%$ confident of attracting at least 1 male, estimated from Eq. (10) as a function of the density of searching males $(\rho)$. Other parameter values: $N=5 ; E=10^{10}$; $\alpha=0.05 \mathrm{~m}^{-1} ; T=1800 \mathrm{~s} ; D=0.002 \mathrm{~m} ; V=0.015 \mathrm{~m} \mathrm{~s}^{-1}$

$$
D_{N N}=\sqrt[3]{\frac{1.41}{\rho}}
$$

If we take the lowest estimate for Sternoptyx diaphana $\left(\rho=2.3 \times 10^{-5} \mathrm{~m}^{-3}\right)$, then this translates into a $D_{N N}$ value of $39 \mathrm{~m}$. This is close to double the largest value quoted by Herring (2000). Thus, our model predicts that for the range of average nearest neighbour distances considered by Herring (2000), a female is highly likely to obtain a mate within $2 \mathrm{~h}$ of beginning bioluminescent signalling. However, to explore this further, we will now explore how the predictions of Fig. 3 are influenced by variation in parameter values. Fig. 4a illustrates that for the relatively slow swimming speed used in our model, the probability of passing through the detection zone without detecting the female is low, and so the predictions of the model are relatively insensitive to the period of the signal $(T)$. Fig. $4 \mathrm{~b}$ demonstrates a similar lack of sensitivity to change in swimming speed. This is not surprising as although increasing swimming speed increases the frequency with which a male encounters detection zones, it also increases the probability that he will pass through it before the female flashes a signal. In fact, this second effect is slightly stronger, so detection times increase slightly with increasing searching speed. In contrast, Fig. 4c shows that small increases in eye diameter greatly reduce discovery times. This occurs because an increase in $D$ leads to an increase in $R$, which both hastens the discovery of a given female's detection zone and decreases the likelihood of passing through the detection zone without detecting a flash.
Similar reasoning explains why increasing the intensity of the signals $(E)$, decreasing the number of photons required for detection $(N)$ or decreasing the beam attenuation coefficient of water $(\alpha)$ all have a substantial effect in reducing detection times (Fig. 4d-f).

Lastly, we could consider male strategies to minimise energy investment rather than minimising time. There are a number of published relations for the cost of transport for swimming fish $C$ (based either on theoretical arguments or on empirical measurements over a range of species), of which perhaps the most commonly cited one is that of Ware (1978):

$$
C=1.17 M^{1.44} V^{2.42}
$$

where $C$ is in Watts, $M$ is the mass in $\mathrm{kg}$ and $v$ is the speed in $\mathrm{m} \mathrm{s}^{-1}$. For a $60 \mathrm{~mm}$ long Sternoptyx diaphana, we use the same weight wet estimate as before, $4 \mathrm{~g}$. The average time to find a female is simply $(Z A)^{-1}$; thus, the average amount of work done to find a female $(W)$ is given by:

$$
W=\frac{C}{Z A}
$$

Fig. 5 shows that because $C$ increases so steeply with increasing speed, the reduced time over which this cost is paid for a faster-moving male does not compensate for the increased power required, and the total energetic cost of finding a female always increases with swimming speed.

\section{DISCUSSION}

The key conclusion of this paper is that the combination of bioluminescent signalling and realistic levels of male mobility should allow mate finding within a small number of hours of a female beginning to signal. Hence, mate finding may not seriously restrict reproductive success in species that can exploit this signalling system. A female can select the time and place most advantageous for egg release then start egg hydration, confident of obtaining a male within a small number of hours of beginning to signal. Such confidence is essential, since egg hydration is generally an irreversible process, and eggs are either lost or absorbed if spawning does not take place immediately afterwards. Hydration times are generally of the order of 6 to 12 h (e.g. Clarke 1987).

In one respect, our model is likely to underestimate detection times; this stems from the assumption of a spherical detection zone. This assumption was necessary to preserve any analytic tractability, as the frequency distribution of chord lengths can only be expressed in a simple closed form (like Eq. 2) for a small number of very simple shapes. However, this 


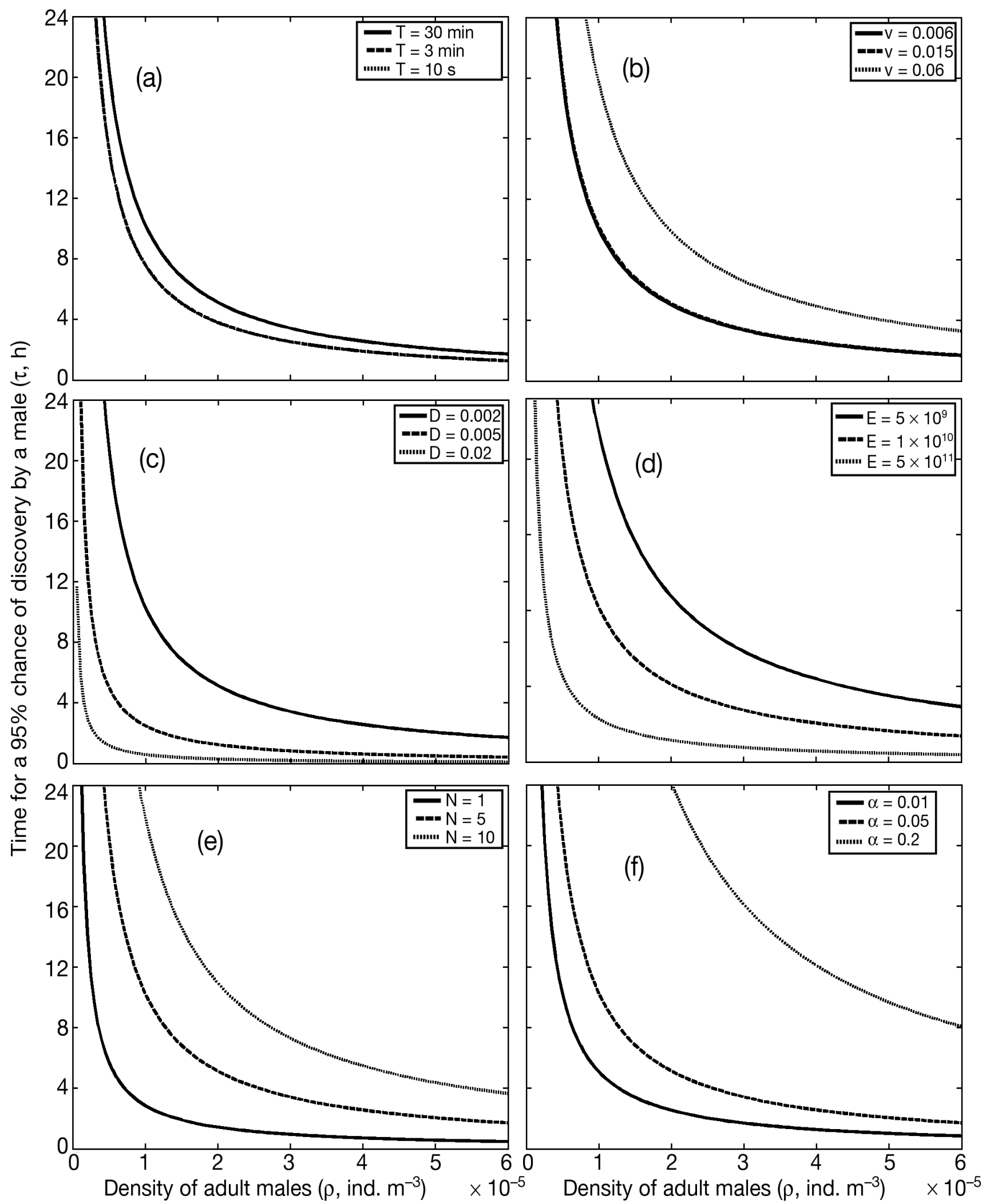

Fig. 4. Estimated time $(\tau)$ for which a female would have to signal to be $95 \%$ confident of attracting at least 1 male, estimated from Eq. (10) as a function of the density of searching males $(\rho)$, and (a) the period of flashing $(T)$, (b) the male search speed $(v)$, (c) the male's pupil diameter $(D)$, (d) the flash strength $(E)$, (e) the number of photons needed for detection $(N)$ and (f) the beam attenuation coefficient of the water $(\alpha)$. Default parameter values: $N=5 ; E=10^{10} ; \alpha=0.05 \mathrm{~m}^{-1}, T=1800 \mathrm{~s}, D=0.002 \mathrm{~m}, V=0.015 \mathrm{~m} \mathrm{~s}{ }^{-1}$ 


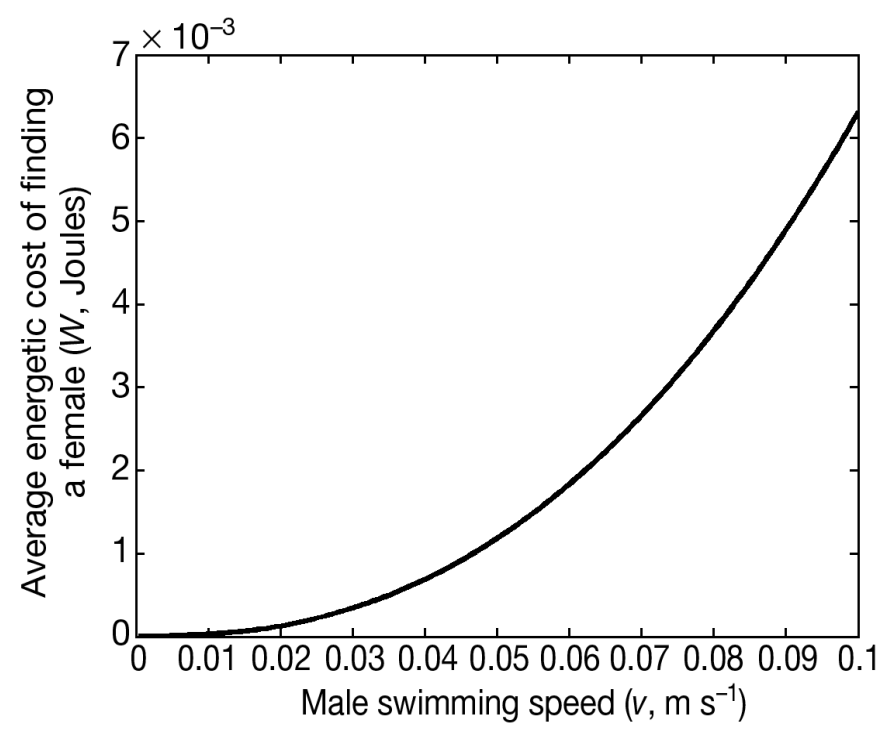

Fig. 5. Average extra energy invested in finding a female $(W)$ as a function of swimming speed $(\mathrm{v})$ for a $4 \mathrm{~g}$ fish, calculated from Eq. (13). Other parameter values: $N=5 ; E=10^{10}$; $\alpha=0.05 \mathrm{~m}^{-1} ; T=1800 \mathrm{~s} ; D=0.002 \mathrm{~m} ; v=0.015 \mathrm{~m} \mathrm{~s}^{-1} ; \rho=$ $3 \times 10^{-5}$ ind. $\mathrm{m}^{-3}$

assumption requires not only that the background is isotropic, but that the light emitted by the female is also. It further assumes that the male can see equally well in all directions. All these requirements are open to challenge. An isotropic background can only be justified in water sufficiently deep that daylight penetration is negligible. Photophores that act to provide crypsis through counter-illumination are likely to provide a highly directional beam, directed downwards parallel to daylight; in contrast, we would expect photophores designed for the type of signalling envisaged in this paper to be evolved to produce a spatially widespread signal. Likewise, we would expect such signalling to influence the evolution of eye design in males to produce as wide a field of vision as possible; detection of predators should produce a similar selection pressure. Notice here that we require the male only to detect light, not the more challenging task of resolving a sharp image. However, for both signal emission and detection, the physical structure of fish is likely to make our assumption of perfect isotropy only an approximation to reality. Although we feel that it may well be a relatively good approximation, it is likely to lead to underestimation of encounter times. However, this concern should be balanced against the likelihood that our estimation of maximum detection distance $(R)$ is very conservative. A more recent formulation of our Eq. (6) suggests that the value for $N$ may in actuality be much lower than we assumed, and hence $R$ may be several times larger than we have assumed (Warrant \& Locket 2004). Since the rate at which the male enters female's detection zones varies with $R^{2}$ and the likeli- hood of such entrances leading to flash detection also increases with $R$, a substantial increase in $R$ could very significantly reduce our predicted detection times. However, there is a pressing need for empirical measurement of naturally occurring flashes and organisms' reactions to nearby flashes before these issues can be fully resolved.

The frequency with which an individual fish produces bioluminescent flashes has been very infrequently reported, even for shallow-water fish (but see Mensinger 1995). However, our simulations make the prediction that we expect flash rates used by deep-sea fish in mate finding to be much lower than the several times a second reported for fireflies and for shallowwater species using bioluminescence for prey finding. Since the extra predation risk and extra energetic expense of flashing more frequently than once every few minutes brings very little improvement in encounter rates, 1 clear prediction from our model is that such slow signalling rates are likely to be a hallmark of bioluminescent mate attraction in the deep sea. This prediction invites empirical investigation. Of these 2 factors (predators and energy), we expect predation to be the dominant factor influencing flashing behaviour, since our calculations suggest that the energy required to produce bioluminescent flashes for mate attraction is relatively trivial, even for organisms with the low metabolisms typical of deep-sea fishes. We also predict that flash rates should increase if males swim significantly faster than considered here. This is highly unlikely on energetic grounds, except perhaps for fish substantially larger than those considered in this study. More plausible is that flash rates will be higher if $R$ is reduced significantly from the values considered here. This would occur if the water had a high particle load (increasing the absorption of light, modelled by an increase in $\alpha$ ), if there is significant background light emanating from other bioluminescent organisms (increasing $N$ ), if the males have particularly small eyes (decreasing $D$ ), or if the females have weak bioluminescent signals (decreasing $E$ ).

Our model only deals with the time taken by a male to detect a bioluminescent flash by a female. It is important to remember that the male must then identify the female as being of the same species and then find his way to the female; neither of these is a trivial exercise. We might speculate that both of these processes might be facilitated by the male responding to his initial detection of a flash by bioluminescent signalling himself, with a view to initiating a signalling dialogue between the 2 fish (analogous to those observed in fireflies: e.g. Lloyd 1977) in order to aid species identification and precise localisation of the female. We would expect this dialogue to feature higher flash rates by the female (aiding localisation) 
and species-specific flash patterns (aiding species recognition).

A key component of our model is the estimation of the distance at which bioluminescent signals can be detected, based on the physical arguments of Warrant (2000). Although the values obtained using Warrant's equation are similar those estimated in previous works (such as those of Baird \& Jumper 1995 and Herring 2000), they are slightly higher. As Baird \& Jumper (1995) discussed at length, the predictions of models such as that presented here are highly sensitive to changes in the value of this parameter. Hence, there is mounting need for us to overcome the challenges associated with testing this component of the model empirically.

Another novel set of predictions generated by the model relates to the searching speed of the males. Although increasing searching speed increases the rate at which males encounter the detection zones around females, it also increases their risk of passing through such a zone without detecting a female. Hence, the time taken to find a female decreases relatively slowly with increasing search speed. However the power required to swim increases very rapidly with increasing speeds. Hence, from an energetic point of view, more total energy would be required to find a female if the searching is carried out at high speed. Hence, from a purely energetic point of view, we would expect males to move relatively sedately (less than 1 body-length $\mathrm{s}^{-1}$ ) when searching for mating opportunities.

Consider now the effectiveness of chemical and light signals for mate attraction in deep-sea fish. As Herring (2000) and others have argued, in terms of distance over which the signal can be detected, chemical communication has an advantage. Jumper \& Baird (1991) provide a simulation model for mate finding through olfaction in deep-sea fish. When parameterised for a specific species of deep-sea hatchetfish, the model predicted that a single pulse of pheromone release could be detected to a maximum range of $100 \mathrm{~m}$. Hence, chemical signals can give an order of magnitude improvement in detection distance over light signals, and would certainly be required for fish that live at low densities and which are relatively immobile. However, notice that this detection range advantage need not translate into reduced time for a female to obtain a male. Although males were relatively similar in Jumper \& Baird's (1991) simulations to those presented here $\left(\rho=3 \times 10^{-5} \mathrm{~m}^{-3} ; v=0.015 \mathrm{~m} \mathrm{~s}^{-1}\right)$, they did not find females dramatically quicker, with Jumper \& Baird (1991) reporting that 'in less than 2 hrs, the probability of detection reached $90 \%$ '. The reason for this is that although a chemical signal can travel greater distances than light signals, they take appreciable time to do so. It required $9 \mathrm{~h}$ for the chemical signal to reach the maximum distance of $100 \mathrm{~m}$ in the simulation results of Jumper \& Baird (1991).
One advantage of a light-based signal is that the female can instantaneously desist from signalling and so greatly reduce the ability of other males of her own species and predators to detect her. In contrast, a female cannot destroy a chemical signal once she has emitted it, rather the signal is finally degraded by chemical and physical processes beyond the female's control. This is relevant in a number of contexts. Having found a male with which she is happy to mate, a female signalling with light can immediately cease signalling, reducing the predation risk to the pair and the risk of being harassed by other males. In order to achieve the same effect, a chemically signalling female and her chosen male must travel away from her original position, such that although the signal remains it no longer provides accurate information about the female's location. Although this movement brings benefits to the mated pair, it has the unattractive sideeffect that males will waste time following olfactory signals only to find no female at the end of their search. This is bad for females that are signalling but have yet to obtain a mate, as well as for males, since it reduces the effective density of males searching freely if a significant fraction of them are investing time and energy in following outdated signals.

A similar advantage to light signalling can be seen in an anti-predatory context. If a female detects a predator nearby (or some other change in her perceived predation risk), then she can instantaneously desist from signalling and so immediately reduce her predation risk. A chemical signaller does not have this option; its only option to reduce the predation risk from its signal is to flee the scene. However, this movement may make it more easy for predators to detect. Further, beginning signalling again from a new location incurs a significant time penalty for a chemically signalling female because of the significant time taken for the chemicals to spread out in the water. No such penalty exists for light signallers. Hence, these arguments suggest that where male motility allows bioluminescent signalling, this may have some advantages over chemical-based signalling. These advantages are particularly likely to be important where females need to retain flexibility of movement (say for anti-predatory reasons), where perceived predation risk can change over short timescales or where harassment of mating pairs by satellite males could be significant.

We emphasise again that bioluminescent and chemical signalling are not mutually exclusive. Chemical signals can be detected at much longer ranges that bioluminescent ones. However, if the male detected the chemical signal released by the female at a considerable distance from her, then following that chemical signal back to find the female's location might be very challenging. This challenge occurs because, as the 
chemical spreads out spatial gradients in chemical intensity that would otherwise give directional information become more diffuse and so harder to detect. Further, measurement of spatial gradients becomes more difficult since smooth gradients become increasingly disrupted by small-scale eddies caused by bulk water movement during the spreading of the chemical signal (see Baird et al. 1996). Hence, one plausible scenario is that chemical signals can act to alert males to the presence of a receptive female without giving good information about her exact whereabouts. This could still be a valuable signal for otherwise quiescent males that can react to this by increasing their movement rates so as to increase their chance of coming closer to the signalling female, where final location could occur by shorter-range detection methods (such as the bioluminescence discussed here). However, if males actively move about their environment, then no such biochemical trigger to activity is required, and here we have demonstrated that bioluminescent signalling alone could be highly effective in facilitating mate finding in a small number of hours for such species.

Acknowledgements. D.M.B. was supported by a Marie Curie Outgoing International Fellowship (MOIF-CT-2004-509286). This manuscript benefited from the perceptive comments of 3 referees. We thank J. Wagner for advice on Sternoptyx anatomy.

\section{LITERATURE CITED}

Alexander RM (1999) Energy for animal life. Oxford University Press, Oxford

Baird RC, Jumper GY (1995) Encounter models and deep-sea fishes: numerical simulations and mate location problem in Sternoptyx diaphana (Pisces, Sternophychidae). DeepSea Res I 42:675-696

Baird RC, Johari H, Jumper GY (1996) Numerical simulation of environmental modulation of chemical signal structure and odor dispersal in the open ocean. Chem Senses 21:121-134

Bradbury JW, Vehrencamp SL (1998) Principles of animal behaviour. Sinauer Associates, Sunderland, MA

Clarke GL, Hubbard CJ (1959) Quantitative records of the luminescent flashing of oceanic animals at great depths. Limnol Oceanogr 4:163-180

Clarke TA (1987) Fecundity and spawning frequency in the Hawaiian anchovy or nehu Encrasicholina purpurea. Fish B-NOAA 85:127-138

Denny MW (1993) Air and water: the biology and physics of life's media. Princeton University Press, Princeton, NJ

Denton EJ (1990) Light and vision at depths greater than 200 m. In: Herring PJ, Campbell AK, Whitfield M, Maddock L (eds) Light and life in the sea. Cambridge University Press, Cambridge, p 127-148

Drazen JC (2002) Energy budgets and feeding rates of Corphaenoides acrolepis and C. armatus. Mar Biol 140:677-686

Gerritsen J, Strickler JR (1977) Encounter probabilities and community structure in zooplankton: a mathematical model. J Fish Res Board Can 34:73-82

Hammerstein P, Parker GA (1987) Sexual selection: games between the sexes. In: Bradbury JW, Andersson M (eds) Sexual selection: testing the alternatives. John Wiley, New York, p 119-142
Hastings JW, Nealson KH (1981) The symbiotic luminous bacteria. In: Starr MP, Stolp H, Truper HG, Balows A, Schkegel HG (eds) Prokaryotes: a handbook of habitats, isolation and identification bacteria. Springer-Verlag, New York, p 1332-1345

Herring PJ (1983) The spectral characteristics of luminous marine organisms. Proc R Soc Lond B 220:183-217

Herring PJ (2000) Species abundance, sexual encounter and bioluminescent signalling in the deep sea. Phil Trans R Soc Lond B 355:1273-1276

Herring PJ (2002) The biology of the deep ocean. Oxford University Press, Oxford

Howell WH, Krueger WH (1987) Family Sternoptychidae, marine hatchetfishes and related species. In: Gibbs $\mathrm{RH}$, Krueger WH (eds) Biology of midwater fishes of the Bermuda Ocean Acre. Smithsonian Institute Press, Washington, DC, p 32-50

Howland HC, Merola S, Basarab JR (2004) The allometry of scaling of the size of vertebrate eyes. Vision Res 44:2043-2065

Johnsen S, Sosik HM (2003) Cryptic coloration and mirrored sides as camouflage strategies in near-surface pelagic habitats: implications for foraging and predator avoidance. Limnol Oceanogr 48:1277-1288

Johnsen S, Widder EA, Mobley CD (2004) Propagation and perception of bioluminescence: factors affecting the success of counterillumination as a cryptic strategy. Biol Bull (Woods Hole) 207:1-16

Jokisch DW, Patton PW, Rajon DA, Inflis BA, Bolch WE (2001) Chord distributions across 3D digital images of a human thoracic vertebra. Med Phys 28:1493-1504

Jumper GY, Baird RC (1991) Location by olfaction: a model and application to the mating problem in the deep-sea hatchetfish Argyropelecus hemigymnus. Am Nat 138:1431-1458

Kornilova GN (1980) Feeding in yellowfin tuna (Thunnus albacares) and Bigeye tuna (Thunnus obesus) in the equatorial zone of the Indian Ocean. J Ichthyol 20:111-119

Land MF, Nilsson DE (2002) Animal eyes. Oxford University Press, Oxford, p 48-49

Lloyd JE (1977) Bioluminescence and communication. In: Sebeok TA (ed) How animals communicate. Indiana University Press, Bloomington, IN, p 164-183

Mackie GO, Mills CE (1983) Use of the Pisces IV submersible for zooplankton studies in coastal waters of British Columbia. Can J Fish Aquat Sci 40:763-776

Mensinger AF (1995) Ecomorphological adaptations to bioluminescence in Porichthys notatus. Environ Biol Fish 44: 133-143

Mensinger AF, Case JF (1990) Luminescent properties of deep sea fish. J Exp Mar Biol Ecol 144:1-15

Mensinger AF, Case JF (1997) Luminescent properties of fishes from nearshore Californian basins. J Exp Mar Biol Ecol 210:75-90

Nelson PA, Zamzow JP, Losey GD (2001) Ultraviolet blocking in the ocular humors of the teleost fish Acanthocybium solandri (Scombridae). Can J Zool 79:1714-1718

Nunes-Halldorson V da S, Duran NL (2003) Bioluminescent bacteria: lux genes as environmental biosensors. Braz J Microbiol 34:91-96

Torres JJ, Belman BW, Childress JJ (1979) Oxygen consumption of midwater fishes as a function of depth of occurrence. Deep-Sea Res 26A:185-197

Ware DM (1978) Bioenergetics of pelagic fish. J Fish Res Board Can 35:220-228

Warrant E (2000) The eyes of deep-sea fishes and the changing nature of visual scenes with depth. Phil Trans R Soc Lond B 355:1155-1159

Warrant EJ, Locket NA (2004) Vision in the deep sea. Biol Rev 79:671-712

Submitted: November 1, 2004; Accepted: February 22, 2005

Proofs received from author(s): May 9, 2005 